\title{
Evaluation of Penalized-Likelihood Estimation Reconstruction on a Digital Time-of-Flight PET/CT Scanner for ${ }^{18}$ F-FDG Whole-Body Examinations
}

\author{
Elin Lindström ${ }^{1,2}$, Anders Sundin ${ }^{1}$, Carlos Trampal $^{3}$, Lars Lindsjö ${ }^{3}$, Ezgi Ilan ${ }^{1,2}$, Torsten Danfors ${ }^{1}$, Gunnar Antoni $^{4}$, \\ Jens Sörensen ${ }^{1,3}$, and Mark Lubberink ${ }^{1,2}$ \\ ${ }^{1}$ Radiology and Nuclear Medicine Division, Department of Surgical Sciences, Uppsala University, Uppsala, Sweden; ${ }^{2}$ Department of \\ Medical Physics, Uppsala University Hospital, Uppsala, Sweden; ${ }^{3}$ PET Centre, Uppsala University Hospital, Uppsala, Sweden; and \\ ${ }^{4}$ Molecular Imaging Division, Department of Medicinal Chemistry, Uppsala University, Uppsala, Sweden
}

The resolution and quantitative accuracy of PET are highly influenced by the reconstruction method. Penalized-likelihood estimation algorithms allow for fully convergent iterative reconstruction, generating a higher image contrast than ordered-subsets expectation maximization (OSEM) while limiting noise. In this study, a type of penalized reconstruction known as block-sequential regularized expectation maximization (BSREM) was compared with time-offlight OSEM (TOF OSEM). Various strengths of noise penalization factor $\beta$ were tested along with various acquisition durations and transaxial fields of view (FOVs) with the aim of evaluating the performance and clinical use of BSREM for ${ }^{18} \mathrm{~F}-\mathrm{FDG}$ PET/CT, both quantitatively and in a qualitative visual evaluation. Methods: Eleven clinical whole-body ${ }^{18} \mathrm{~F}-\mathrm{FDG}$ PET/CT examinations acquired on a digital TOF PET/CT scanner were included. The data were reconstructed using BSREM with point-spread function recovery and $\beta$-factors of $133,267,400$, and 533 -and using TOF OSEM with point-spread function-for various acquisition times per bed position and various FOVs. Noise level, signal-to-noise ratio (SNR), signal-to-background ratio (SBR), and SUV were analyzed. A masked evaluation of visual image quality, rating several aspects, was performed by 2 nuclear medicine physicians to complement the analysis. Results: The lowest levels of noise were reached with the highest $\beta$-factor, resulting in the highest SNR, which in turn resulted in the lowest SBR. A $\beta$-factor of 400 gave noise equivalent to TOF OSEM but produced a significant increase in $\operatorname{SUV}_{\max }(11 \%)$, SNR (22\%), and SBR (12\%). BSREM with a $\beta$-factor of 533 at a decreased acquisition duration (2 min/bed position) was comparable to TOF OSEM at a full acquisition duration ( $3 \mathrm{~min} / \mathrm{bed}$ position). Reconstructed FOV had an impact on BSREM outcome measures; SNR increased and SBR decreased when FOV was shifted from 70 to $50 \mathrm{~cm}$. The evaluation of visual image quality resulted in similar scores for reconstructions, although a $\beta$-factor of 400 obtained the highest mean whereas a $\beta$-factor of 267 was ranked best in overall image quality, contrast, sharpness, and tumor detectability. Conclusion: In comparison with TOF OSEM, penalized BSREM reconstruction resulted in an increased tumor $S U V_{\max }$ and an improved SNR and SBR at a matched level of noise. BSREM allowed for a shorter acquisition than TOF OSEM, with equal image quality.

Received Aug. 22, 2017; revision accepted Jan. 25, 2018.

For correspondence or reprints contact: Elin Lindström, Uppsala University Hospital, SE-75185 Uppsala, Sweden.

E-mail: elin.lindstrom@surgsci.uu.se

Published online Feb. 15, 2018.

COPYRIGHT (C 2018 by the Society of Nuclear Medicine and Molecular Imaging.
Key Words: PET/CT; FDG; image reconstruction; penalization factor; block-sequential regularized expectation maximization

J Nucl Med 2018; 59:1152-1158

DOI: 10.2967/jnumed.117.200790

\section{$\mathbf{P}$} noninvasive quantitative imaging of physiologic and pathologic processes in vivo. The quantitation accuracy of tracer uptake is affected by several factors, including the transformation of collected emission data into reconstructed images. This study investigated the performance of a new penalized-likelihood reconstruction algorithm and its impact on image quality and quantification of tracer accumulation (SUV), as compared with the standard nonregularized ordered-subsets expectation maximization (OSEM).

Statistical iterative reconstruction methods such as maximumlikelihood expectation maximization were first mentioned in the late 1970s $(1,2)$, and OSEM, a modified expectation maximization method, has been used for clinical image reconstruction for more than $20 \mathrm{y}$ (3). In contrast to analytic methods, there are no closedform solutions available using statistical approaches. The algorithm must be stopped before convergence is reached in order to avoid image degradation due to excessive noise. However, stopping before convergence leads to a bias in the final image estimate toward the initial estimate, as well as a nonuniform convergence in which large volumes of high tracer activity converge more rapidly than adjacent small volumes and low-activity areas. Penalizedlikelihood expectation maximization allows for fully convergent iterative reconstruction, leading to higher image contrast than for OSEM while limiting noise.

Block-sequential regularized expectation maximization (BSREM) (Q.Clear; GE Healthcare) is a penalized-likelihood estimation reconstruction algorithm recently developed to improve quantitation accuracy while maintaining high image quality (Eq. 1) (4). By adding a penalty function to the objective function, the algorithm enforces conditions on the image estimate that allow for full convergence and edge preservation (Eq. 2) (5,6). Fully convergent iterative methods require excess computational time, but the block-sequential approach reduces computational time (3). Previous shortcomings of penalized reconstruction methods, such as blocky artifacts and patchy images, are overcome by activity-dependent smoothing and 
spatially modulated penalty parameters. The image estimates using BSREM are based on priors, and the algorithm can be expressed by the following objective function:

$$
\phi(x, y)=L(x, y)-\beta R(x),
$$

where $x$ is the image estimate based on PET measurements $y, L$ is the Poisson log likelihood, and $R$ describes the relative-difference penalty function (7):

$$
R(x)=\sum_{j=1}^{n_{y}} \sum_{k \in N_{j}} w_{j} w_{k} \frac{\left(x_{j}-x_{k}\right)^{2}}{x_{j}+x_{k}+\gamma\left|x_{j}-x_{k}\right|} .
$$

In Equation 2, $N_{j}$ is a set of neighbors of the $j^{\text {th }}$ voxel, $w_{j}$ and $w_{k}$ are weights of the relative-difference function, and $\gamma$ in the potential function controls edge preservation. The $\beta$-factor is a positive regularization parameter that controls the trade-off between noise level and resolution and is the only user-input variable in the commercially available version of the Q.Clear software (8).

BSREM has been shown to provide better quantitation accuracy than OSEM in phantom studies and clinical studies with simulated lesions (9-11). Lesion detectability with BSREM has also been found to be higher than or equal to that with $\operatorname{OSEM}(11,12)$. A few evaluations of visual image quality have been conducted, and they showed that images acquired with BSREM are considered equal to or better than images acquired with $\operatorname{OSEM}(9,13)$. A recent observer study investigated the clinical performance of BSREM with 4 different $\beta$-factors of between 300 and 400 (13). A combined phantom and observer study found a $\beta$-factor of 400 to be the optimal penalization factor based on signal-to-background ratio (SBR). A semiquantitative and visual analysis was performed with a $\beta$-factor of 400 for diagnosis of mediastinal lymph nodes and pulmonary nodules and found that SBR, signal-to-noise ratio (SNR), and $\mathrm{SUV}_{\max }$ increased compared with OSEM, although no improvement in accuracy could be seen $(9,14,15)$.

In the present study, the performance of BSREM was investigated for 4 different penalization factors $\beta$ within the range of $133-533$ to evaluate clinical use for whole-body ${ }^{18} \mathrm{~F}-\mathrm{FDG}$ PET/CT on a digital time-of-flight (TOF) PET/CT scanner. The primary aim was to compare quantitative results with the standard reconstruction method, TOF OSEM with point-spread function (PSF) recovery, together with a visual assessment of image quality by 2 observers. The secondary aim was to investigate the effect of different transverse fields of view (FOVs) and image acquisition times per bed position on BSREM.

\section{MATERIALS AND METHODS}

\section{Data Acquisition}

PET Scanner. All scans were performed on a Discovery MI PET/ CT device (GE Healthcare). This TOF-capable scanner has lutetium yttrium oxyorthosilicate detectors coupled to silicon photomultipliers, with axial and transaxial FOVs of 20 and $70 \mathrm{~cm}$, respectively, producing 71 image planes with a $2.79-\mathrm{mm}$ slice thickness. The TOF resolution of the scanner is approximately $370 \mathrm{ps}$, sensitivity is $14 \mathrm{cps} / \mathrm{kBq}$, and intrinsic spatial resolution is about $4.0 \mathrm{~mm}$ at the center of the FOV (16).

National Electrical Manufacturers Association (NEMA) Phantom. A NEMA image quality phantom with sphere diameters of 10, 13, 17, 22,28 , and $37 \mathrm{~mm}$ was used (17). The phantom background was filled with approximately $20 \mathrm{MBq}$ of ${ }^{18} \mathrm{~F}$, and the spheres were filled with a 4 times higher radioactivity concentration. The phantom was scanned until 100 million prompt counts were acquired.
Clinical Data. Data from the 3-dimensional whole-body ${ }^{18} \mathrm{~F}-\mathrm{FDG}$ PET/CT scans of 11 patients were included in this study. For 8 oncologic patients, the intravenously administered activity was $3.7 \pm$ $0.2 \mathrm{MBq} / \mathrm{kg}$ (mean $\pm \mathrm{SD}$; range, 3.6-4.0 MBq/kg) and the uptake time between administration and imaging was $67 \pm 6$ min (range, 58-75 $\mathrm{min}$ ). For the remaining 3 patients, who were imaged because of suspected inflammatory disease, the administered activity was $5.9 \pm 0.1 \mathrm{MBq} / \mathrm{kg}$ (range, $5.8-5.9 \mathrm{MBq} / \mathrm{kg}$ ) and the uptake time was $187 \pm 5 \mathrm{~min}$ (range, 183-194 min). All PET/CT examinations were performed from the proximal femur to the base of the skull with an acquisition time of $3 \mathrm{~min}$ per bed position $(\mathrm{min} / \mathrm{bp})$. The images were anonymized before reconstruction and further analysis and were not used for clinical patient diagnosis; this study was therefore not covered by the Swedish law on ethics review for research in humans, and no application to the ethics review board was required.

\section{Image Reconstruction}

NEMA Phantom. Images were reconstructed using TOF OSEM ( 3 iterations, 16 subsets, and a 5-mm gaussian postprocessing filter) with PSF compensation, as recommended by the manufacturer for standard clinical image reconstruction, and also using TOF BSREM including PSF (from here on referred to as BSREM) with $\beta$-factors of 133, 267, 400, and 533. A 50-cm FOV was used with a $256 \times 256$ matrix, resulting in a $1.95 \times 1.95 \times 2.79 \mathrm{~mm}$ pixel size.

Clinical Data. For each patient's PET/CT examination, 20 reconstructions were produced for analysis using the manufacturer's software (version Columbia PET.96). PET images were reconstructed in the same manner as for the phantom, with a transaxial FOV of both 50 and $70 \mathrm{~cm}$, resulting in a nearly isotropic pixel size of $2.73 \times 2.73 \times$ $2.79 \mathrm{~mm}$ for the $70-\mathrm{cm}$ FOV. In addition to the $3 \mathrm{~min} / \mathrm{bp}$ reconstruction, list-mode files were rebinned using only the first 2 and $1 \mathrm{~min} / \mathrm{bp}$ and reconstructed again using the $70-\mathrm{cm}$ FOV.

\section{Image Analysis}

NEMA Phantom. Using in-house-developed software in MATLAB (MathWorks), regions of interest (ROIs) were automatically placed to include the spheres, background, and lung insert of the phantom images, as specified by the NEMA protocol. In addition, spheric volumes of interest (VOIs) with diameters identical to each sphere were placed to include the spheres.

Background variability as a measure of image noise was defined as the SD of the activity concentration in all individual voxels in the largest background ROIs located $\pm 4 \mathrm{~cm}$ from the plane containing the sphere centers, divided by the mean activity of these background ROIs. Contrast-to-noise ratio (CNR) was calculated as contrast recovery (Eq. 3) divided by background variability, and lung residual correction error was calculated as the mean counts in the lung VOI divided by the mean counts in the background ROIs.

$$
\text { Contrast recovery }=\frac{\frac{C_{H}}{C_{B}}-1}{\frac{a_{H}}{a_{B}}-1},
$$

where $C$ is counts and $a$ is activity in hot and background ROIs (subscript $H$ and $B$, respectively).

Clinical Data. Images were analyzed using the PET/CT Review tool on an Advantage Workstation (AW 4.7 extension 8; GE Healthcare). Level of noise was defined as SD normalized to $\mathrm{SUV}_{\text {mean }}$ of a large spheric reference VOI placed in normal liver. SNR was calculated as lesion $\mathrm{SUV}_{\max }$ divided by noise level, and SBR was calculated as lesion $\mathrm{SUV}_{\text {max }}$ divided by $\mathrm{SUV}_{\text {mean }}$ of the liver reference. Lesions were delineated using a $41 \%$ threshold of the maximum voxel value in the TOF OSEM image; a bookmark containing the location of the delineated lesion was then propagated to the BSREM 


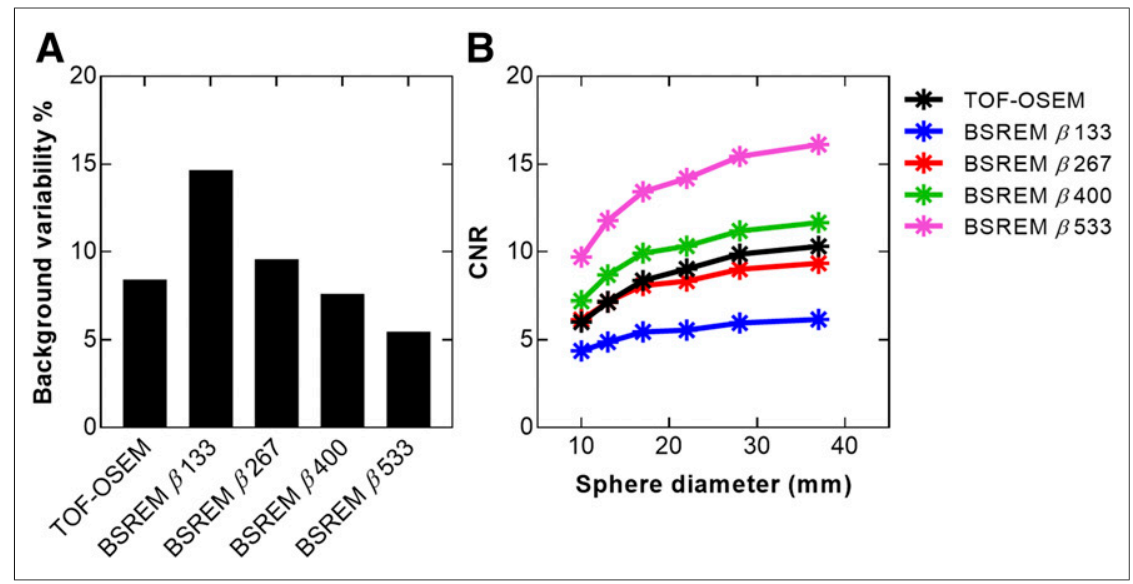

FIGURE 1. NEMA image quality phantom reconstruction data showing background variability (A) and CNR as function of sphere diameter (B). Reconstruction methods were TOF OSEM (3 iterations, 16 subsets, 5 -mm gaussian postprocessing filter, and PSF) and BSREM (including TOF and PSF) with $\beta$-factors of $133,267,400$, and 533 and $50-\mathrm{cm}$ FOV.
0.05. Spearman rank correlation $(\rho)$ was used to determine the association between different parameters.

\section{RESULTS}

\section{NEMA Phantom}

Phantom results are displayed in Figure 1. The residual correction error of the lung was lower for all BSREM $\beta$-factors than for TOF OSEM ( $\sim 2.5 \%$ vs. $7 \%$, respectively). Background variability increased with decreasing $\beta$-factor, whereas CNR decreased with decreasing sphere diameter and decreasing $\beta$-factor. The difference in CNR between reconstruction methods increased with increasing sphere diameter: With a $\beta$-factor of more than 267, CNR was higher for BSREM than for TOF OSEM for all spheres, with $61 \%$ higher CNR for a $\beta$-factor of 533 for the 10-mm sphere (CNR: 9.7 vs. 6.0 for BSREM vs. TOF OSEM, respectively). reconstructed images, creating new VOIs using $41 \%$ thresholding. In this study, the optimal $\beta$-factor was defined in terms of yielding a noise level equivalent to that of TOF OSEM.

Visual Evaluation. The quantitative analysis was supplemented by a visual evaluation of image quality by 2 nuclear medicine physicians with more than $20 \mathrm{y}$ of experience in diagnostic ${ }^{18} \mathrm{~F}-\mathrm{FDG}$ PET. Ten examinations were assessed, each including 5 reconstructions (TOF OSEM and BSREM with $\beta$-factors of $133,267,400$, and 533). Before assessment, all data were anonymized with regard to patient identity and reconstruction method. The 3-dimensional PET image sets were evaluated one by one in a randomized order and scored per an arbitrary 4-grade scale (poor, moderate, good, and very good) for 7 different parameters (overall image quality, contrast, sharpness, presence of artifacts, noise level, homogeneity of liver background, and tumor detectability). A first case was used as a training case and read by both reviewers in consensus. All PET examinations in the visual evaluation were reconstructed using a $50-\mathrm{cm}$ FOV and $3 \mathrm{~min} / \mathrm{bp}$. A coregistered intravenous contrast-enhanced CT examination was available with each reconstruction to provide an anatomic reference.

Statistical Analysis. A paired Student $t$ test was used to investigate differences in assessment scores between reconstruction methods. Statistical significance was considered present for $P$ values of less than

\section{Clinical Data}

Eleven clinical PET/CT examinations with 25 lesions in total were included in the analysis. The mean lesion size was $9.6 \pm$ $15.6 \mathrm{~cm}^{3}$ (range, $0.5-69.4 \mathrm{~cm}^{3}$ ), with an $\mathrm{SUV}_{\max }$ of $11.2 \pm 6.6$ (range, 2.6-28.4), measured in TOF OSEM images with a $3 \mathrm{~min} / \mathrm{bp}$ acquisition and a $70-\mathrm{cm}$ FOV. The mean liver reference volume was $19.2 \pm 0.6 \mathrm{~cm}^{3}$ (range, $18.0-19.6 \mathrm{~cm}^{3}$ ), with an $\mathrm{SUV}_{\text {mean }}$ of $2.1 \pm$ 0.3 (range, 1.6-2.6) (Table 1).

The choice of penalization factor influenced the image both visually and quantitatively as shown by the example in Figure 2. The lowest level of noise was reached with the highest $\beta$-factor, resulting in the highest SNR and in turn the lowest SBR. Compared with TOF OSEM, BSREM with a $\beta$-factor of 400 produced-in reconstructions with a 70-cm FOV and a 3-min acquisition-a mean lesion $\mathrm{SUV}_{\max }$ that was increased by $11 \%$ (from 11.2 to 12.2), a mean SNR that was increased by $22 \%$ (from 99.3 to 120.6), and a mean SBR that was increased by $12 \%$ (from 5.4 to 6.0) (Fig. 3). Applying an $\mathrm{SUV}_{\text {mean }}$ threshold of greater than 3 resulted in more lesions detected with BSREM than with TOF OSEM, regardless of $\beta$-factor (24 lesions with BSREM with $\beta$-factors of 133, 267, 400, and 533, vs. 21 lesions with TOF OSEM).

TABLE 1

Quantitative Measures of Reference Sphere in Healthy Liver Tissue Using TOF OSEM and BSREM

\begin{tabular}{|c|c|c|c|c|c|}
\hline \multirow[b]{2}{*}{ Measure } & \multirow[b]{2}{*}{ TOF OSEM* } & \multicolumn{4}{|c|}{ BSREM } \\
\hline & & $\beta$-factor, 133 & $\beta$-factor, 267 & $\beta$-factor, 400 & $\beta$-factor, 533 \\
\hline Volume $\left(\mathrm{cm}^{3}\right)$ & $19.2(18.0-19.6)$ & & & & \\
\hline$S_{\text {SUax }}$ & $2.9(2.1-4.0)$ & $4.2(2.7-5.4)$ & $3.1(2.2-4.0)$ & $2.8(2.0-3.7)$ & $2.6(2.0-3.5)$ \\
\hline$S_{S U V}$ mean & $2.1(1.6-2.6)$ & $2.0(1.6-2.5)$ & $2.0(1.6-2.5)$ & $2.1(1.6-2.6)$ & $2.1(1.6-2.6)$ \\
\hline $\mathrm{SUV}_{\mathrm{SD}}$ & $0.2(0.2-0.4)$ & $0.4(0.3-0.6)$ & $0.3(0.2-0.4)$ & $0.2(0.2-0.3)$ & $0.2(0.1-0.3)$ \\
\hline Noise level ${ }^{*}$ & $0.11(0.09-0.16)$ & $0.22(0.18-0.28)$ & $0.13(0.11-0.17)$ & $0.10(0.08-0.13)$ & $0.09(0.07-0.11)$ \\
\hline
\end{tabular}

${ }^{*}$ Measured in liver and defined as SUV $\mathrm{SD}$ divided by SUV $\mathrm{mean}_{\text {. }}$

Data are mean followed by range. TOF OSEM used 3 iterations, 16 subsets, 5-mm gaussian postprocessing filter, and PSF. BSREM included TOF and PSF with $\beta$-factors of $133,267,400$, and $533 ; 70-\mathrm{cm}$ FOV; and $3 \mathrm{~min} / \mathrm{bp}$. 


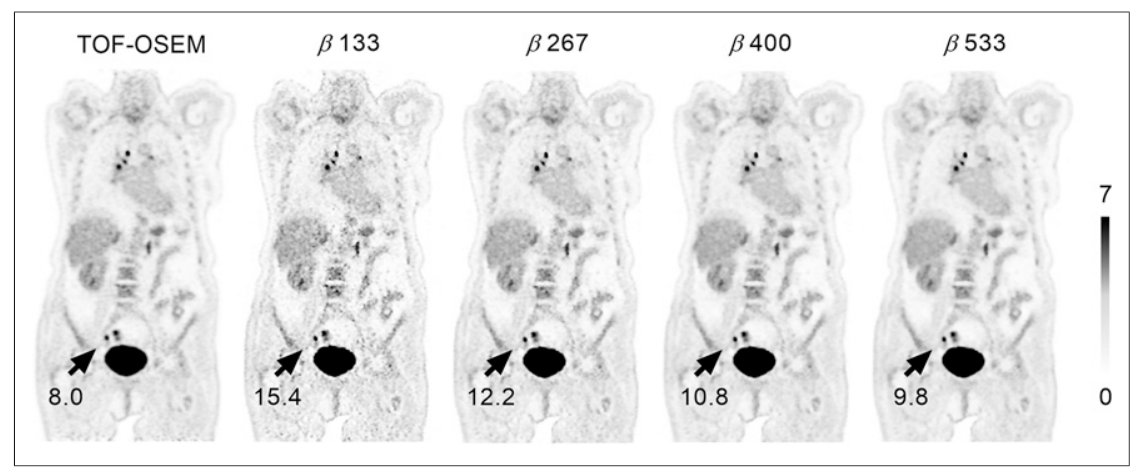

FIGURE 2. Coronal whole-body ${ }^{18} \mathrm{~F}-\mathrm{FDG}$ PET images of patient with inflammatory disease, demonstrating ovarian uptake (arrows) by $\mathrm{SUV}_{\max }$ obtained with respective reconstruction method. Images were reconstructed with $70-\mathrm{cm}$ FOV and $3 \mathrm{~min} / \mathrm{bp}$ acquisition, using TOF OSEM (3 iterations, 16 subsets, $5-\mathrm{mm}$ gaussian postprocessing filter, and PSF) and BSREM (including TOF and PSF) with $\beta$-factors of $133,267,400$, and 533.

Lesion Size Dependence. In comparisons of BSREM with TOF OSEM, the relative difference in $\mathrm{SUV}_{\max }$ was generally larger for smaller lesions, irrespective of $\beta$-factor. The mean increase in SUV $_{\max }$ for BSREM with a $\beta$-factor of 400 was $16 \%$ for lesions with volume of less than $3 \mathrm{~cm}^{3}$ and $8 \%$ for a volume of more than $3 \mathrm{~cm}^{3}$, and the trend was similar for SNR $(26 \%$ and $18 \%$, respectively) and SBR (17\% and 9\%, respectively). There was a small negative association between percentage increase in $\mathrm{SUV}_{\max }$ and lesion volume over the entire cohort $(\rho=-0.3)$.

Acquisition Duration. Visual and quantitative differences in various acquisition times are exemplified in Figure 4. Decreasing the acquisition from 3 to $2 \mathrm{~min} / \mathrm{bp}$ changed the optimal $\beta$-factor to 533 to reach a noise level equivalent to that of $3 \mathrm{~min} / \mathrm{bp}$ TOF OSEM images while maintaining a higher SNR (Fig. 5). For BSREM with a $\beta$-factor of 533 at a $2 \mathrm{~min} / \mathrm{bp}$ acquisition duration, $\mathrm{SUV}_{\text {max }}$ increased by $9 \%$, SNR by $12 \%$, and SBR by $10 \%$, compared with TOF OSEM at a full acquisition duration. The noise level was higher than in $3 \mathrm{~min} / \mathrm{bp}$ TOF OSEM images for all $\beta$-factors using a $1 \mathrm{~min} / \mathrm{bp}$ acquisition.

FOV Dependence. Quantitative measures using TOF OSEM were not affected by a change in reconstructed FOV from 70 to $50 \mathrm{~cm}$, as opposed to BSREM, for which SNR increased and SBR decreased. Background noise decreased with a smaller FOV, giving a noise level equivalent to that of TOF OSEM with a $\beta$-factor of 267 instead of a $\beta$-factor of 400 . The trade-off between SNR and noise, and between SBR and noise, using different FOVs followed the same curvatures (Fig. 6). At noise equivalence, there was an increase in both SNR and SBR for BSREM compared with TOF OSEM.

Visual Evaluation. Reconstruction using BSREM with a $\beta$-factor of 267 was ranked highest in the observers' assessment concerning overall image quality, whereas BSREM with a $\beta$-factor of 400 obtained a higher total mean for all aspect scores (Fig. 7; Supplemental Table 1; supplemental materials are available at http://jnm. snmjournals.org). Images with lower $\beta$-factors were considered to have good contrast but a high level of noise compared with TOF OSEM and higher $\beta$-factors. TOF OSEM was ranked higher than BSREM with $\beta$-factors of 400 and 533 regarding contrast and sharpness but lower regarding noise level and liver background. BSREM with $\beta$-factors of 267,400 , and 533 were regarded as better than TOF OSEM in terms of tumor detectability. The evaluation resulted in overall minor differences between reconstruction methods, not only in the total score but also within each category. Interobserver agreement was good for the presence of artifacts and tumor detectability but lower for scoring contrast, whereas interobserver agreement concerning reconstruction method was best for TOF OSEM and a $\beta$-factor of 267 and worst for a $\beta$-factor of 133 . The total mean score of both observers ranked a $\beta$-factor of 133 as the least preferred reconstruction, with TOF OSEM next to last, and a $\beta$-factor of 400 as most favored.

\section{DISCUSSION}

This study addressed the relationship between noise level and resolution in BSREMreconstructed images and evaluated the optimal algorithm settings for clinical diagnosis in oncologic patients and patients with inflammatory or infectious disease. This relatively new algorithm has been investigated previously, but not for data acquired on a digital TOF PET/CT scanner and not while varying the acquisition time and FOV. The digital silicon photomultiplier detector technology and improved timing resolution of the Discovery MI result in improved sensitivity and count
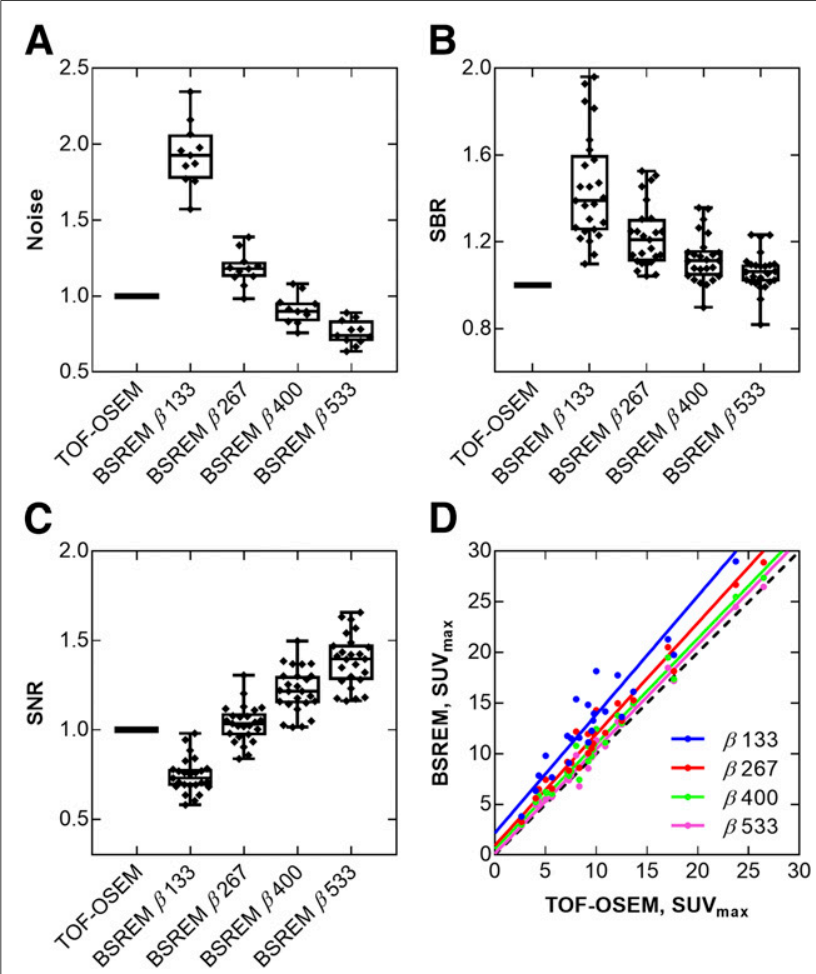

D

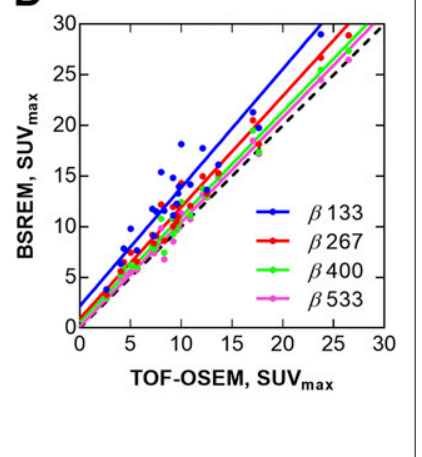

FIGURE 3. Quantitative data from ${ }^{18} \mathrm{~F}-\mathrm{FDG}$ PET/CT examinations of 11 patients with total of 25 lesions. Box plots show noise level (A), SBR (B), and SNR (C) for different reconstruction methods, and correlation

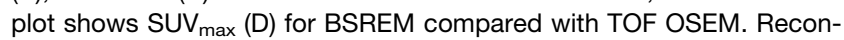
struction methods were TOF OSEM ( 3 iterations, 16 subsets, $5-\mathrm{mm}$ gaussian postprocessing filter, and PSF) and BSREM (including TOF and PSF) with $\beta$-factors of $133,267,400$, and $533 ; 70-\mathrm{cm} \mathrm{FOV;} \mathrm{and}$ $3 \mathrm{~min} / \mathrm{bp}$ acquisition. All data were normalized to data obtained by TOF OSEM. Dashed line represents line of identity. 


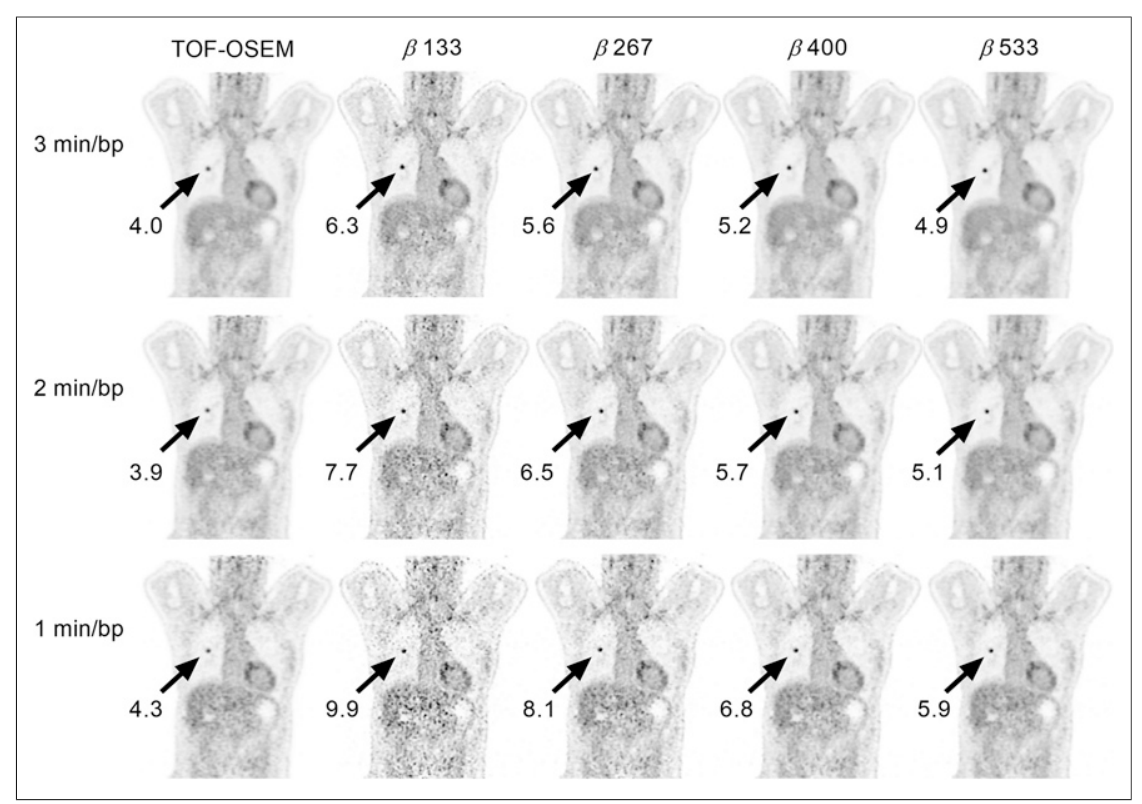

FIGURE 4. Coronal whole-body ${ }^{18} \mathrm{~F}$-FDG PET images of patient with adenocarcinoma, demonstrating SUV $V_{\max }$ of lesion (arrows) obtained with respective reconstruction method (gray scale 0 6). Images were reconstructed with 70-cm FOV using TOF OSEM (3 iterations, 16 subsets, $5-\mathrm{mm}$ gaussian postprocessing filter, and PSF) and BSREM (including TOF and PSF) with $\beta$-factors of 133, 267, 400, and 533 for 3, 2, and $1 \mathrm{~min} / \mathrm{bp}$ acquisitions.

rate statistics compared with conventional PET/CT systems $(16,18,19)$. This investigation showed that it is challenging to specify one $\beta$-factor that is overall optimal. The choice of $\beta$-factor depends on whether the primary requirement is shorter acquisition, lower radiation dose, higher image quality, or better lesion depiction. In clinical PET/CT imaging, all these factors need to be considered and harmonized to generate, on the one hand, the best possible image quality and, on the other hand, a low radiation dose to the patient and reasonable acquisition times to achieve a swift workflow.

BSREM was compared with TOF OSEM using 3 iterations, 16 subsets, and a 5-mm gaussian postprocessing filter. Evaluation of TOF OSEM with a larger number of iterations or a narrower filter would have led to a higher SBR and higher noise levels, and correspondingly to noise equivalent to BSREM for lower $\beta$-factors. However, we chose to use the settings recommended by the manufacturer as our reference, and further optimization of OSEM was outside the scope of this work. Nevertheless, to illustrate the effect of a narrower filter or more iterations in TOF OSEM, we included some additional data in Figure 6. This shows that the signal obtained using TOF OSEM will never increase to match that of BSREM without a simultaneous increase in noise to a higher level than for BSREM.

The range of $\beta$-factors-from 133 to 533 in steps of 133 - chosen in this work may seem somewhat peculiar and in need of further explanation. These values were based on the implementation of BSREM in the Discovery MI pilot reconstruction software, for which $\beta$-factors were initially $50 \%$ too high in terms of background noise, compared with $\beta$-factors used on other GE Healthcare systems. The $\beta$-factors were corrected in the final release of the software, so that a $\beta$-factor of 200 in the pilot software translated to 133 in the final software, and so on. Our initially chosen $\beta$-factors of $200-800$ in steps of 200 thus translated into $133-533$ in steps of 133 .

The phantom study showed both similarities to and differences from the clinical image assessment. In the phantom study, the lowest background variability and highest CNR were for BSREM with a $\beta$-factor of 533; however, for clinical image reading, noise level and SNR were optimal for a $\beta$-factor of 533, whereas a $\beta$-factor of 133 resulted in the best SBR. For clinical whole-body ${ }^{18} \mathrm{~F}-\mathrm{FDG}$ PET/CT imaging purposes, BSREM with a $\beta$-factor of 400 , resulting in a high SNR, a high SBR, and a noise level like that of TOF OSEM with a 5-mm gaussian postprocessing filter, would probably be the optimal choice, also considering the EANM guidelines suggesting a maximum coefficient of variation below $15 \%$, which is consistently reached only for $\beta$-factors of 400 and higher (20).

In the visual evaluation, 7 image quality criteria were assessed using a 4-grade arbitrary scale. The scoring methods of previous studies vary considerably in number of quality criteria and grading scales. Usually, merely a few grades are applied for each image criterion, but a 100-grade slide-ruler scale has also been used (9,13-15). In the present study, a 4-grade scale was, however, considered sufficient to avoid ranking difficulties. Ranking of all PET examinations for each patient, from best to worst, constitutes an alternative scoring method that was not considered appropriate for the present study.

The 2 observers were masked to patient identity and order of reconstruction method but were well aware of the principles of BSREM and the influence of the $\beta$-factor on the resulting images. When assessing a new technique in imaging, there is always an inherent problem in that the observers are trained to read images reconstructed with a well-known technique and applying standard parameters. Consequently, the observers in the present study were 


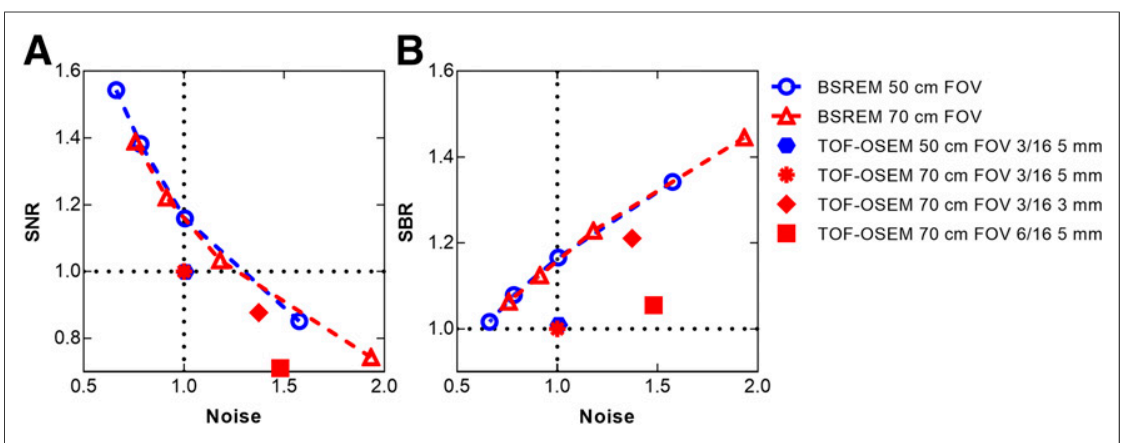

FIGURE 6. Trade-off curves showing SNR vs. noise (A) and SBR vs. noise (B) for reconstructions using TOF OSEM ( 3 iterations, 16 subsets, $5-\mathrm{mm}$ gaussian postprocessing filter, and PSF) and BSREM (including TOF and PSF) with $\beta$-factors of 133, 267, 400, and 533 and transaxial FOV of $50 \mathrm{~cm}$ (blue) and $70 \mathrm{~cm}$ (red). Decreasing $\beta$-factor is seen with increasing noise for BSREM data points in both plots. All data were normalized to TOF OSEM with $70-\mathrm{cm}$ FOV, and data points represent mean values. For reference, additional reconstructions with $70-\mathrm{cm}$ FOV using TOF OSEM with PSF and 3 iterations, 16 subsets, and 3-mm gaussian postprocessing filter, and TOF OSEM with PSF and 6 iterations, 16 subsets, and 5-mm gaussian postprocessing filter, are also shown in both graphs.

well acquainted with TOF OSEM images and might therefore have been biased and rated these higher than the BSREM images. All PET/CT examinations had been performed on patients in whom disseminated cancer with clearly ${ }^{18} \mathrm{~F}-\mathrm{FDG}$-avid metastasesor inflammatory or infectious disease with clearly ${ }^{18} \mathrm{~F}-\mathrm{FDG}$-avid foci-had been diagnosed on PET/CT with a high level of confidence. Possible differences in image quality grading between reconstruction methods might therefore have been more evident had the evaluation been done using images from patients with smaller and less ${ }^{18} \mathrm{~F}-\mathrm{FDG}$-avid lesions. Furthermore, a group larger than the 11 patients in the present study might also have increased these differences. Analysis of a larger number of tumor lesions over a wider volume range could also have improved the results concerning SUV lesion volume dependence. The results of

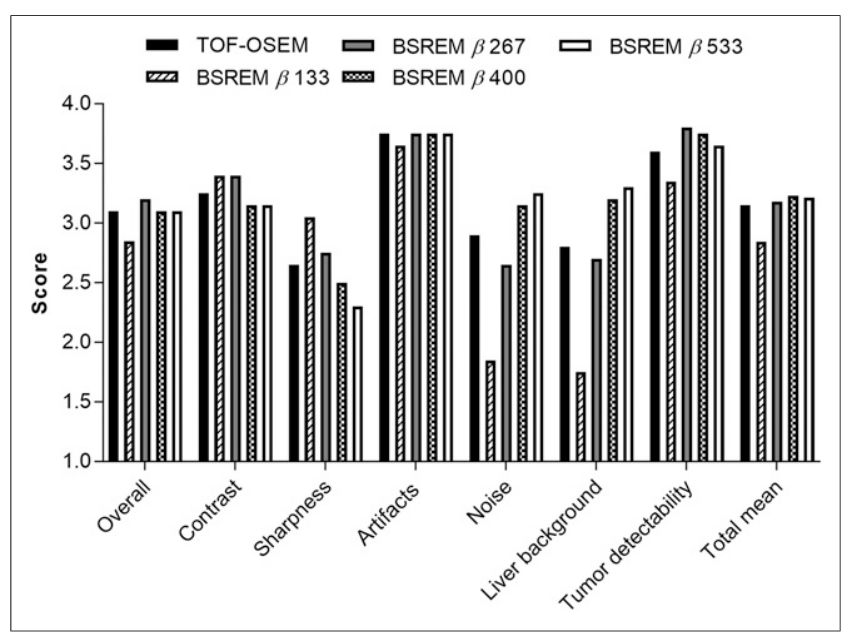

FIGURE 7. Visual scoring of image quality by 2 observers per arbitrary scale (1, poor; 2 , moderate; 3 , good; 4 , very good). Seven different aspects of image quality were considered, and last category in plot represents summarized total mean score. There were 5 sets of images for each of 10 patients. Reconstruction used TOF OSEM ( 3 iterations, 16 subsets, 5-mm gaussian postprocessing filter, and PSF) and BSREM (including TOF and PSF) with $\beta$-factors of 133, 267, 400, and 533; $50-\mathrm{cm} \mathrm{FOV}$, and $3 \mathrm{~min} / \mathrm{bp}$ acquisition. this study were, however, in concordance with previous study results $(11,12,14,15)$.

A shorter image acquisition is preferable in terms of both patient comfort and patient throughput, allowing for more PET/CT examinations per day. This study showed that the acquisition can be reduced from 3 to $2 \mathrm{~min} / \mathrm{bp}$ when BSREM is used instead of TOF OSEM, for a mean saving of $5 \mathrm{~min}$ for a scan of 5 bed positions. Reducing the acquisition to $1 \mathrm{~min} / \mathrm{bp}$ would, however, require a higher $\beta$-factor than was tested in the present study to result in noise levels similar to those of TOF OSEM. Another option is to lower the administered activity instead of shortening the acquisition, allowing for reduced radiation doses, which would be especially favorable for younger patients.

Image noise increased when the transaxial FOV was extended, causing a shift to the right in the trade-off curves illustrating the relationship between SNR and noise as well as SBR and noise (Fig. 6). The shift can be related to the specific penalty modulation of the BSREM algorithm (Steve Ross of GE Healthcare, written communication, December 2017).

Differences in uptake and biodistribution between PET tracers might influence the choice of $\beta$-factor. The optimal settings of the BSREM algorithm for whole-body ${ }^{18}$ F-FDG PET/CT may therefore not necessarily produce the desired results for PET/CT with other tracers and should therefore be evaluated separately for each PET tracer in a clinical setting. Further future research should also investigate the relation between intensity variations in dynamic PET acquisitions and SUV measurements.

\section{CONCLUSION}

In comparison with TOF OSEM, penalized-likelihood BSREM reconstruction resulted in an increased tumor $S_{U} V_{\max }$ and an improved SNR and SBR at a matched level of noise. BSREM allowed for a shorter acquisition per bed position than TOF OSEM, with equal image quality.

\section{DISCLOSURE}

This work was partly financed by research collaboration between Uppsala University Hospital and GE Healthcare. No other potential conflict of interest relevant to this article was reported.

\section{ACKNOWLEDGMENTS}

We acknowledge the team at the Uppsala University Hospital PET Centre for their assistance in image acquisition, reconstruction, and data handling. We also thank Tarik Cengiz, Dr. Charles Stearns, and Dr. Steve Ross from GE Healthcare for discussions, assistance, and support.

\section{REFERENCES}

1. Dempster AP, Laird NM, Rubin DB. Maximum likelihood from incomplete data via the EM algorithm. J R Stat Soc Series B Stat Methodol. 1977;39:1-38.

2. Shepp LA, Vardi Y. Maximum likelihood reconstruction for emission tomography. IEEE Trans Med Imaging. 1982;1:113-122. 
3. Hudson HM, Larkin RS. Accelerated image reconstruction using ordered subsets of projection data. IEEE Trans Med Imaging. 1994;13:601-609.

4. Ross S. Q.Clear White Paper. Chicago, IL: GE Healthcare; 2014.

5. de Pierro AR, Yamagishi MEB. Fast EM-like methods for maximum 'a posteriori' estimates in emission tomography. IEEE Trans Med Imaging. 2001;20:280-288.

6. Ahn S, Fessler JA. Globally convergent image reconstruction for emission tomography using relaxed ordered subsets algorithm. IEEE Trans Med Imaging. 2003;22:613-626.

7. Nuyts J, Bequé D, Dupont P, Mortelmans L. A concave prior penalizing relative differences for maximum-a-posteriori reconstruction in emission tomography. IEEE Trans Nucl Sci. 2002;49:56-60.

8. Nuyts J, Fessler J. A penalized-likelihood image reconstruction method for emission tomography, compared to postsmoothed maximum-likelihood with matched spatial resolution. IEEE Trans Med Imaging. 2003;22:1042-1052.

9. Teoh EJ, McGowan DR, Macpherson RE, Bradley KM, Gleeson FV. Phantom and clinical evaluation of the Bayesian penalized likelihood reconstruction algorithm Q.Clear on an LYSO PET/CT system. J Nucl Med. 2015;56: 1447-1452.

10. Ahn S, Ross S, Asma E, et al. Quantitative comparison of OSEM and penalized likelihood image reconstruction using relative difference penalties for clinical PET. Phys Med Biol. 2015;60:5733-5751.

11. Wangerin KA, Wollenweber S, Ross SG, et al. Evaluation of lesion detectability in positron emission tomography when using a convergent penalized likelihood image reconstruction method. J Med Imaging (Bellingham). 2017;4:011002.

12. Parvizi N, Franklin JM, McGowan DR, Teoh EJ, Bradley KM, Gleeson FV. Does a novel penalized likelihood reconstruction of ${ }^{18} \mathrm{~F}$-FDG PET-CT improve signalto-background in colorectal liver metastases? Eur J Radiol. 2015;84:1873-1878.
13. Sah BR, Stolzmann P, Delso G, et al. Clinical evaluation of a block sequential regularized expectation maximization reconstruction algorithm in ${ }^{18} \mathrm{~F}-\mathrm{FDG}$ PET/ CT studies. Nucl Med Commun. 2017;38:57-66.

14. Teoh EJ, McGowan DR, Bradley KM, et al. ${ }^{18} \mathrm{~F}-\mathrm{FDG}$ PET/CT assessment of histopathologically confirmed mediastinal lymph nodes in non-small cell lung cancer using a penalised likelihood reconstruction. Eur Radiol. 2016;26:4098-4106.

15. Teoh EJ, McGowan DR, Bradley KM, Belcher E, Black E, Gleason FV. Novel penalized likelihood reconstruction of PET in the assessment of histologically verified small pulmonary nodules. Eur Radiol. 2016;26:576-584.

16. Hsu DFC, Ilan E, Peterson WT, Uribe J, Lubberink M, Levin CS. Studies of a next generation silicon-photomultiplier-based time-of-flight PET/CT system. J Nucl Med. 2017;58:1511-1518.

17. National Electrical Manufacturers Association. Performance Measurement of Positron Emission Tomographs. Rosslyn, VA: National Electrical Manufacturers Association; 2012. NEMA standards publication NU 2-2012.

18. van der Vos CS, Koopman D, Rijnsdorp S, et al. Quantification, improvement, and harmonization of small lesion detection with state-of-the-art PET. Eur J Nucl Med Mol Imaging. 2017;44:4-16.

19. Nguyen NC, Vercher-Conejero JL, Sattar A, et al. Image quality and diagnostic performance of a digital PET prototype in patients with oncologic diseases: initial experience and comparison with analog PET. J Nucl Med. 2015;56: 1378-1385

20. Boellaard R, Willemsen AT, Arends B, Visser EP. EARL procedure for assessing PET/CT system specific patient FDG activity preparations for quantitative FDG PET/CT studies. EANM research Ltd. (EARL) website. http://earl.eanm. $\mathrm{org} / \mathrm{html} / \mathrm{img} / \mathrm{pool} / \mathrm{EARL}$-procedure-for-optimizing-FDG-activity-for-quantitativeFDG-PET-studies_version_1_1.pdf. Updated April 4, 2013. Accessed April 10, 2018. 